\title{
Survey on: Privacy Strategy Presumption for Users Content on Sharing Sites
}

\author{
Nilesh N. Sahastrabuddhe ${ }^{1}$, N. D. Kale ${ }^{2}$ \\ ${ }^{1}$ Department of Computer Engineering, PVPIT, Bavdhan Pune, India \\ ${ }^{2}$ Professor, Assistant Professor, Department of computer Engineering, PVPIT, Bavdhan Pune, India
}

\begin{abstract}
Usage of social media's has been considerably increasing no of pictures clients share through social destinations, keeping up protection has turned into a major issue, as per recent studies there are incidents where user accidently share the personal information sites like Facebook, twitter.For this reason there is need of tool which control the access of shared content. To overcome this problem, we propose an Adaptive Privacy Policy Prediction (A3P) framework to help clients make security settings for their pictures. To achieve this goal system access user preferences and image content and metadata information. We propose a two-level system which determines the best policy of user's history and user's image metadata which is being upload on the site. The proposed algorithm automatically generates the privacy prediction for each new image.
\end{abstract}

Keywords: Social media, Adaptive Privacy Policy Prediction, metadata

\section{Introduction}

Pictures are in no time one of the key enabling operators of customers' accessibility. Sharing happens both among in advance settled social occasions of known people or gatherings of companions (e. g., Google+, Flickr or Picasa), besides dynamically with people outside the customers gatherings of companions, for explanations behind social revelation to help them perceive new partners and get some answers concerning partners interests and social environment. In any case, semantically rich pictures may reveal content sensitive information [2]. Consider a photo of an understudies 2012 graduation ceremony, for example. It could be shared inside a Google+ circle or Flickr total, however may unnecessarily reveal the students, family members and diverse partners. Sharing pictures inside online substance sharing sites therefore may quickly lead to undesirable disclosure[3] and security encroachment. Propel, the productive method for online media makes it plausible for various customers to assemble rich gathered information about the proprietor of the circulated substance and the subjects in the conveyed substance. The gathered information can achieve unanticipated presentation of one's social environment and provoke to maul of one's near and dear information. Most substance sharing locales allow customers to enter their security slants. Shockingly, late audits have shown that customers fight to set up and keep up such security settings [1][11]. One of the guideline reasons gave is that given the measure of shared information this strategy can be dull and botch slanted. In this way, many have perceived the need of approach proposition structures which can push customers to easily and genuinely outline assurance settings. In any case, existing suggestions for automating insurance settings radiate an impression of being inadequate to address the unique security needs of pictures[3][5] in light of the measure of information irrefutably passed on inside pictures, and their relationship with the online environment wherein they are revealed.

In this wander, we propose an Adaptive Privacy Policy Prediction (A3P) system which arrangements to give customers a trouble free security settings experience by means of normally making tweaked courses of action. The A3P system handles customer exchanged pictures, and considers the going with criteria that effect one's security settings of pictures:

\section{Motivation}

The guideline motivation driving this venture due to sharing pictures inside online substance sharing locales, consequently, may rapidly prompt to undesirable revelation and protection infringement. The tireless way of online media makes it feasible for different clients to gather rich collected data about the proprietor of the distributed substance and the subjects in the distributed substance. The amassed data can bring about startling introduction of one's social surroundings and prompt to manhandle of one's close to home data

\section{Literature Survey}

Jonathan Anderson proposed a worldview called Privacy Suites which permits clients to effortlessly pick suites" of protection settings. A security suite can be made by a specialist utilizing protection programming. Protection Suites could likewise be made specifically through existing design UIs or sending out them to the dynamic organization. The protection suite is circulated through existing conveyance channels to the individuals from the social locales. The weakness of a rich programming dialect is less understandability for end client [7].

FabeahAdu-Oppong created security settings in view of the idea of groups of friends [11]. It gives an electronic answer for ensure individual data. The system named Social Circles Finder, consequently produces the companion's rundown. It is a strategy that examinations the group of friends of a man and distinguishes the power of relationship and in this manner groups of friends give a significant arrangement of companions for setting protection approaches. The application will recognize the groups of friends of the 


\section{International Journal of Science and Research (IJSR) \\ ISSN (Online): 2319-7064}

Index Copernicus Value (2015): 78.96 | Impact Factor (2015): 6.391

subject yet not demonstrate them to the subject. The subject will then be made inquiries about their readiness to share a bit of their own data. In light of the answers the application finds the visual diagram of clients[11].

KambizGhazinour outlined a recommender framework known as Your Privacy Protector that comprehends the social net conduct of their security settings and suggesting sensible protection choices. It uses client's close to home profile, User's interests and User's protection settings on photograph collections as parameters and with the assistance of these parameters the framework develops the individual profile of the client. It naturally learned for a given profile of clients and dole out the protection alternatives. It permits clients to see their present security settings on their interpersonal organization profile, to be specific Facebook, and screens and distinguishes the conceivable protection dangers [16]

Alessandra Mazzia presented PViz Comprehension Tool an interface and framework that relates all the more straightforwardly with how clients show gatherings and security strategies connected to their systems. PViz permits the client to comprehend the perceivability of her profile as per consequently developed, characteristic sub-groupings of companions, and at various levels of granularity. Since the client must have the capacity to recognize and recognize naturally developed gatherings, we additionally address the vital sub-issue of creating powerful gathering names [17].

\section{Problem Statement}

Keeping up protection has turned into a noteworthy issue, as showed by a late flood of advertised episodes where clients unintentionally shared individual data. In light of these episodes, the need of apparatuses to help clients control access to their mutual substance is clear. Towards tending to this need, we propose an Adaptive Privacy Policy Prediction (A3P) framework to help clients create protection settings for their pictures. We inspect the part of social setting, picture substance, and metadata as would be prudent markers of clients' security inclinations. We propose a twolevel system which as indicated by the client's accessible history on the site decides the best accessible protection approach for the client's pictures being transferred.

\section{Existing System}

Most substance sharing sites permit clients to enter their protection inclinations. Tragically, late reviews have demonstrated that clients battle to set up and keep up such security settings. One of the principle reasons gave is that given the measure of shared data this procedure can be repetitive and blunder inclined. In this way, many have recognized the need of arrangement suggestion frameworks which can help clients to effortlessly and legitimately design protection settings. Sharing pictures inside online substance sharing destinations, in this manner, may rapidly prompt to undesirable exposure and protection infringement. Encourage, the persevering way of online media makes it feasible for different clients to gather rich totaled data about the proprietor of the distributed substance and the subjects in the distributed substance. The collected data can bring about unforeseen presentation of one's social surroundings and prompt to mishandle of one's close to home data.

\section{Proposed System}

The A3P framework comprises of two primary segments: A3P-center and A3P-social. The general information stream is the accompanying. At the point when a client transfers a picture, the picture will be first sent to the A3P-center. The $\mathrm{A} 3 \mathrm{P}$-center groups the picture and figures out if there is a need to conjure the $\mathrm{A} 3 \mathrm{P}$-social. By and large, the $\mathrm{A} 3 \mathrm{P}$ center predicts arrangements for the clients specifically in view of their authentic conduct. On the off chance that one of the accompanying two cases is confirmed valid, A3Pcenter will summon A3Psocial: (i) The client does not have enough information for the kind of the transferred picture to direct strategy forecast; (ii) The A3P-center recognizes the late significant changes among the client's group about their protection rehearses alongside client's expansion of long range interpersonal communication exercises (expansion of new companions, new posts on one's profile and so forth).

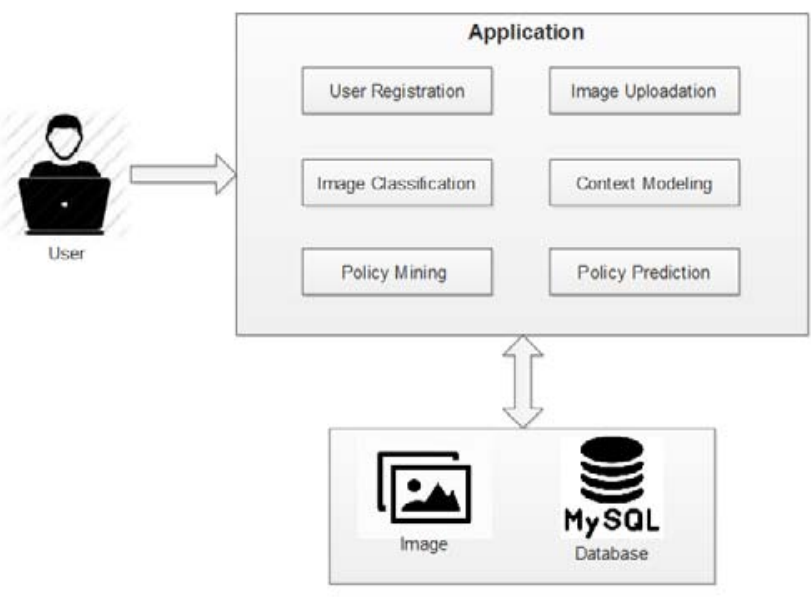

Figure 1: Proposed system architecture

The proposed system consist of following Methodologies,

\section{1) Content-Based Classification}

To get gatherings of pictures that might be connected with comparative protection inclinations, we propose a various leveled picture grouping which orders pictures initially in light of their substance and afterward refine every class into subcategories in view of their metadata. Pictures that don't have metadata will be assembled just by substance. Such a various leveled order gives a higher need to picture content and minimizes the impact of missing labels. Take note of that it is conceivable that a few pictures are incorporated into different classes the length of they contain the ordinary substance elements or metadata of those classifications.

\section{2)Metadata-Based Classification}

The metadata-based arrangement bunches pictures into subcategories under previously mentioned benchmark classifications. The procedure comprises of three principle steps. The initial step is to concentrate catchphrases from the metadata connected with a picture. The metadata considered in our work are labels, subtitles, and remarks. The second step is to determine a delegate hypernym (indicated as h) from every metadata vector. The third step is to discover a 


\section{International Journal of Science and Research (IJSR) \\ ISSN (Online): 2319-7064}

Index Copernicus Value (2015): 78.96 | Impact Factor (2015): 6.391

subcategory that a picture has a place with. This is an incremental technique. Toward the starting, the principal picture frames a subcategory as itself and the agent hypernyms of the picture turns into the subcategory's illustrative hypernyms.

\section{3) Adaptive Policy Prediction}

The strategy expectation calculation gives an anticipated approach of a recently transferred picture to the client for his/her reference. All the more vitally, the anticipated strategy will mirror the conceivable changes of a client's protection concerns. The expectation procedure comprises of three principle stages: (i) policy normalization; (ii) policy mining; and (iii) policy prediction.

\section{4) Scope}

a) To provide users a hassle free privacy settings experience by automatically generating personalized policies.

b) The impact of social environment and personal characteristics. Social context of users, such as their profile information and relationships with others may provide useful information regarding users' privacy preferences. For example, users interested in photography may like to share their photos with other amateur photographers.

c) The role of image's content and metadata. In general, similar images often incur similar privacy preferences, especially when people appear in the images. For example, one may upload several photos of his kids and specify that only his family members are allowed to see these photos

\section{Conclusion}

We have proposed an Adaptive Privacy Policy Prediction (A3P) framework that helps clients computerize the security arrangement settings for their transferred pictures. The A3P framework gives a complete system to surmise protection inclinations in view of the data accessible for a given client. We additionally viably handled the issue of chilly begin, utilizing social setting data. Our exploratory review demonstrates that our $\mathrm{A} 3 \mathrm{P}$ is a viable device that offers noteworthy enhancements over current ways to deal with security.

\section{Acknowledgments}

I take this golden opportunity to owe our deep sense of gratitude to my project guide Prof. N.D.Kale help and valuable guidance with a lot of encouragement throughout this paper work, right from selection of topic work up to its completion. My sincere thanks to Head of the Department of Computer Engineering Prof.Dr.B.K.Sarkar who continuously motivated and guided us for completion of this paper. I am also thankful to our PG Coordinator, all teaching and nonteaching staff members, for their valuable suggestions and valuable co-operation for partially completion of this work. I specially thank to those who helped us directly-indirectly in completion of this work successfully.

\section{References}

[1] A. Acquisti and R. Gross, "Imagined communities: Awareness,information sharing, and privacy on the facebook," in Proc.6th Int. Conf. Privacy Enhancing Technol. Workshop, 2006,pp. 36-58.

[2] R. Agrawal and R. Srikant,"Fast algorithms for mining associationrules in large databases," in Proc. 20th Int. Conf. Very Large DataBases, 1994, pp. 487-499.

[3] S. Ahern, D. Eckles, N. S. Good, S. King, M. Naaman, and R. Nair,"Over-exposed?: Privacy patterns and considerations in onlineand mobile photo sharing," in Proc. Conf. Human Factors Comput.Syst., 2007, pp. 357-366.

[4] M. Ames and M. Naaman, "Why we tag: Motivations for annotationin mobile and online media," in Proc. Conf. Human FactorsComput. Syst., 2007, pp. 971-980.

[5] A. Besmer and H. Lipford, "Tagged photos: Concerns, perceptions, and protections," in Proc. 27th Int. Conf. Extended AbstractsHuman Factors Comput. Syst., 2009, pp. 4585-4590.

[6] D. G. Altman and J. M. Bland "Multiple significance tests: Thebonferroni method," Brit. Med. J., vol. 310, no. $6973,1995$.

[7] J. Bonneau, J. Anderson, and L. Church, "Privacy suites: Sharedprivacy for social networks," in Proc. Symp. Usable Privacy Security,2009.

[8] J. Bonneau, J. Anderson, and G. Danezis, "Prying data out of asocial network," in Proc. Int. Conf. Adv. Soc. Netw. Anal. Mining.,2009, pp.249-254.

[9] H.-M. Chen, M.-H. Chang, P.-C. Chang, M.-C. Tien, W. H. Hsu, and J.-L. Wu, "Sheepdog: Group and tag recommendation forflickr photos by automatic searchbased learning," in Proc. $16^{\text {th }} \mathrm{ACM}$ Int. Conf. Multimedia, 2008, pp. 737-740.

[10] M. D. Choudhury, H. Sundaram, Y.-R. Lin, A. John, and D. D.Seligmann, "Connecting content to community in social mediavia image content, user tags and user communication," in Proc.IEEE Int. Conf. Multimedia Expo, 2009, pp.1238-1241.

[11] L. Church, J. Anderson, J. Bonneau, and F. Stajano, "Privacy stories:Confidence on privacy behaviors through end user programming,"in Proc. 5th Symp. Usable Privacy Security, 2009.

[12]R. da Silva Torres and A. Falc ao, "Content-based image retrieval:Theory and applications," Revista de Inform_aticaTe_orica e Aplicada,vol. 2, no. 13, pp. $161-185,2006$

[13] R. Datta, D. Joshi, J. Li, and J. Wang, "Image retrieval: Ideas, influences, and trends of the new age," ACM Comput. Surv., vol. 40, no.2, p. 5, 2008.

[14] J. Deng, A. C. Berg, K. Li, and L. Fei-Fei, "What does classifyingmore than 10,000 image categories tell us?" in Proc. 11th Eur. Conf.Comput. Vis.: Part V, 2010, pp. 71-84. [Online]. Available: http://portal.acm.org/citation.cfm?id=1888150.1888157

[15] A. Kapadia, F. Adu-Oppong, C. K. Gardiner, and P. P. Tsang, "Social circles: Tackling privacy in social networks," in Proc.Symp. Usable Privacy Security, 2008.

[16] KambizGhazinour, Stan Matwin and Marina Sokolova,"Yourprivacyprotector:A Recommender System For Privacy Settings In Social Networks", 


\section{International Journal of Science and Research (IJSR)}

ISSN (Online): 2319-7064

Index Copernicus Value (2015): 78.96 | Impact Factor (2015): 6.391

International Journal of Security, Privacy and Trust Management ( IJSPTM) Vol 2, No 4, August 2013.

[17] Alessandra Mazzia Kristen LeFevre and Eytan Adar, The PViz Comprehension Tool for Social Network Privacy Settings, Tech. rep., University of Michigan, 2011. 